\title{
Projected Performance Advantage of Multilayer Graphene Nanoribbons as a Transistor Channel Material
}

\author{
Yijian Ouyang $^{1}(\square)$, Hongjie Dai ${ }^{2}$, and Jing Guo ${ }^{1}(\bowtie)$ \\ ${ }^{1}$ Department of Electrical and Computer Engineering, University of Florida, Gainesville, FL 32611, USA \\ ${ }^{2}$ Department of Chemistry, Stanford University, Stanford, CA 94305, USA \\ Received: 2 October 2009 / Revised: 13 November 2009 / Accepted: 15 November 2009 \\ (C) The Author(s) 2010. This article is published with open access at Springerlink.com
}

\begin{abstract}
The performance limits of a multilayer graphene nanoribbon (GNR) field-effect transistor (FET) are assessed and compared with those of a monolayer GNRFET and a carbon nanotube (CNT) FET. The results show that with a thin high dielectric constant (high- $\kappa$ ) gate insulator and reduced interlayer coupling, a multilayer GNRFET can significantly outperform its CNT counterpart with a similar gate and bandgap in terms of the ballistic on-current. In the presence of optical phonon scattering, which has a short mean free path in the graphene-derived nanostructures, the advantage of the multilayer GNRFET is even more significant. Simulation results indicate that multilayer GNRs with incommensurate non-AB stacking and weak interlayer coupling are the best candidates for high-performance GNRFETs.
\end{abstract}

\section{KEYWORDS}

graphene nanoribbon (GNR), multilayer graphene, new channel material, field-effect transistor, carbon nanotube $(\mathrm{CNT})$

\section{Introduction}

Graphene [1-3] is a monolayer of carbon atoms packed into a honeycomb lattice and possesses an atomically thin body and an area that is orders of magnitude greater, making it an ideal two-dimensional (2-D) system. A 2-D graphene is a semimetal without a bandgap, but a bandgap opens if a field-effect transistor (FET) channel is built on a nanometer-wide graphene nanoribbon (GNR) due to the width direction confinement [4]; this also results in subband formation [5]. The high mobility (up to $200000 \mathrm{~cm}^{2} /(\mathrm{V} \cdot \mathrm{s})$ ) and carrier velocity $\left(\sim 10^{8} \mathrm{~cm} / \mathrm{s}\right)$ demonstrated in 2-D graphene have stimulated strong interests in graphene electronics [1]. The transport properties of GNRs in experiments have been so far hindered by imperfect edges $[6,7]$, but excellent transport properties have been theoretically predicted for structurally perfect GNRs [8]. A GNRFET, however, suffers from the problem of a low on-current due to its nanometer-wide channel, and it has been previously shown that the ballistic performance limits of a monolayer GNRFET are no better than a carbon nanotube (CNT) FET in terms of the on-current to on-off current ratio [9].

In this study we assess the performance limits of GNRFETs and CNTFETs using a well-established ballistic transistor model, which has previously been applied to various 2-D and 1-D channel transistors [10]. The performance limits of a transistor are achieved when the contacts are ideal and the channel is ballistic (i.e., no scattering). Schottky barriers can play an important role in CNTFETs [11] and GNRFETs.

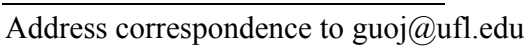


The Schottky barrier is known to lower the on-current and increase the off-current due to ambipolar $I-V$ characteristics. These detrimental effects in Schottky barrier CNTFETs, however, can be eliminated by using a metal-oxide-semiconductor (MOS) FET device structure [12], which has heavily doped source and drain extensions as shown in Fig. 1(a). The performance limits are therefore assessed for a ballistic MOSFET structure with semi-infinite source and drain extensions. Under these conditions, the simple semiclassical model agrees with detailed quantum mechanical transistor simulations for a channel length down to about $10 \mathrm{~nm}$ [10], as long as the direct source-drain tunneling current is relatively small compared to the total source-drain current.

Multilayer GNRs have been demonstrated in recent experiments $[13,14]$. We show that the modeled ballistic performance limits of a multilayer GNRFET can be significantly better than the corresponding CNTFET in terms of the on-current to on-off current ratio. The advantage becomes even bigger in the presence of optical phonon (OP) scattering, which is known to be strong in graphene-derived nanostructures.

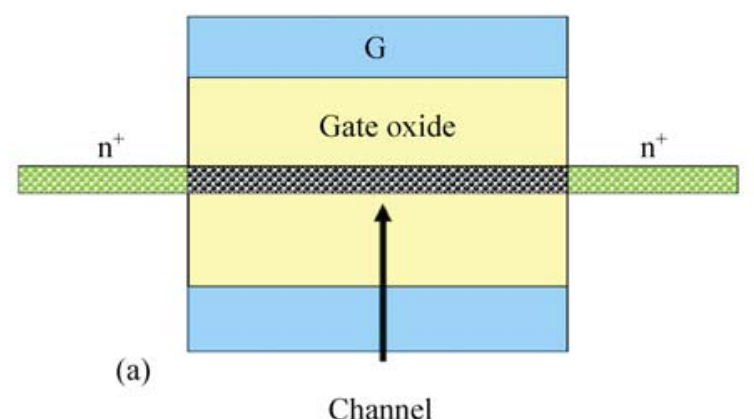

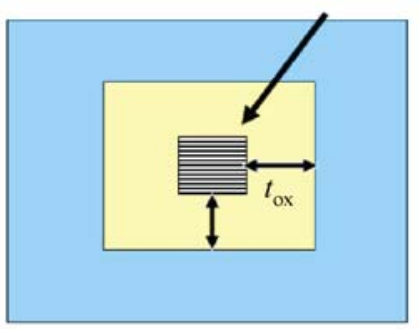

(b)

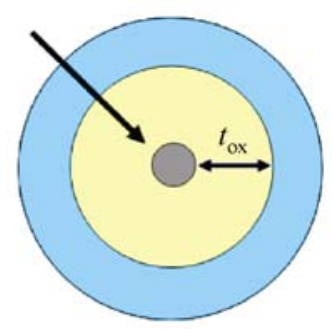

(c)
Figure 1 Schematic structure of the modeled multilayer GNRFET and CNTFET: (a) the MOSFET has an intrinsic channel and heavily doped source and drain extensions; (b) the cross section of the wrapped-around gate GNRFET; (c) the cross section of the CNTFET with a coaxial gate
The important role of gating technology and interlayer coupling are investigated in order to realise the performance advantage of multilayer GNRFETs over CNTFETs. We found that weak interlayer coupling, which is accessible through incommensurate non- $\mathrm{AB}$ stacking, is desired for a high-performance multilayer GNRFET.

\section{Approach}

To ensure a fair comparison, the modeled graphene nanoribbon and carbon nanotube have similar bandgaps. The $(20,0)$ zigzag CNT is semiconducting, which results in a diameter of $d_{\mathrm{CNT}} \approx 1.6 \mathrm{~nm}$ and a bandgap of $E_{\mathrm{g}} \approx 0.50 \mathrm{eV}$. The $n=22$ armchair edge GNR (AGNR) (where $n$ denotes the number of carbon dimer lines [15]) is also semiconducting, which results in a width of $W_{\mathrm{GNR}} \approx 2.7 \mathrm{~nm}$ and a bandgap of $E_{\mathrm{g}} \approx$ $0.52 \mathrm{eV}$ for a monolayer GNR. Both transistors have doped source and drain extensions, as schematically shown in Fig. 1(a). For the GNRFET, a wrapped-around gate is used as shown in Fig. 1(b). For the CNTFET, a coaxial gate is used as shown in Fig. 1(c). The nominal gate insulator is 3-nm-thick $\mathrm{ZrO}_{2}$, which has a relative dielectric constant $\kappa \approx 25$. A power supply voltage of $V_{\mathrm{DD}}=0.5 \mathrm{~V}$ is used.

The bandstructures of the CNT and GNR channels are required as an input to the transistor model. A nearest-neighbor $p_{\mathrm{z}}$ tight binding approach was used. The hopping integral was taken as $t_{\mathrm{cc}}=-2.7 \mathrm{eV}$. The edge effect plays an important role in the GNR, and a factor of 1.12 was used for the hopping parameter between two edge carbon atoms to take account of the edge relaxation effect [16]. For the AB-stacking structure of the multilayer graphene (Fig. 2), an interlayer coupling of $0.3 \mathrm{eV}$ was set only for two vertically aligned atoms in two neighboring layers [17]. The nominal device has an interlayer coupling of zero, which is the lower limit of interlayer coupling. Weak interlayer coupling can be achieved by incommensurate non-AB-stacking, and leads to better device performance as discussed below. The nominal value was also varied in order to examine the effect of interlayer coupling strength on the device performance. 


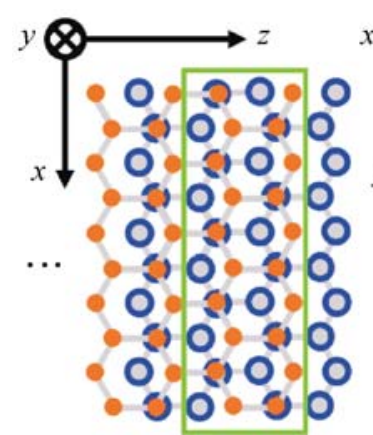

(a)

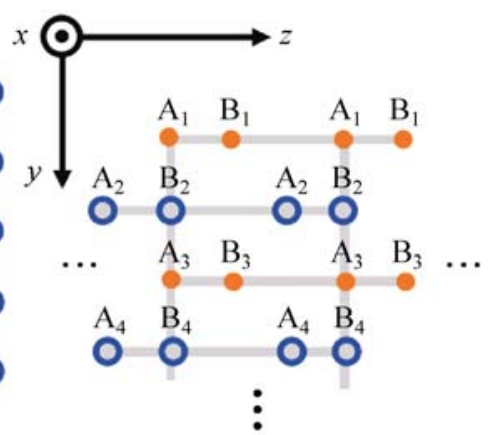

(b)
Figure 2 The atomistic structure of an AB-stacking multilayer GNR. The $z$ direction is defined along the GNR transport direction, the $x$ direction along the width direction, and the $y$ direction along the thickness direction. "A" or " $\mathrm{B}$ " in (b) denotes atoms in A or B sublattices, respectively. The subscript denotes the layer index in a multilayer GNR. The modeled multilayer GNR has armchair edges

A "top-of-barrier" transistor model was used to assess the performance limits of the multilayer GNRFETs. The model fills the $+k$ states at the top of the barrier with the source Fermi level $E_{\mathrm{FS}}$ and the $-k$ states with the drain Fermi level $E_{\mathrm{FD}}$ as shown in Fig. 3(a). Self-consistent electrostatics was treated by a simple capacitance model, in which the gate insulator capacitance value was computed by a numerical Poisson solver and source- (drain-) channel capacitance was ignored for simplicity. The OP scattering in carbon nanotubes [18] or graphene nanoribbons has a short mean free path of $\sim 10 \mathrm{~nm}$, which could play an important role even for transistors with a sub-100 nm channel length. We also considered the transistor performance in the presence of OP scattering by setting an effective Fermi level at $E_{\mathrm{F}}^{\prime}=E_{\mathrm{ES}}-\hbar \omega_{\mathrm{OP}}$ which determines the population of the $-k$ states in the presence of OP scattering, as shown in Fig. 3(b). $\hbar \omega_{\mathrm{OP}} \approx 0.18 \mathrm{eV}$ is the OP energy in CNTs and GNRs.

\section{Results}

Because the transistor performance strongly depends on the bandstructure of the channel material, we first examine the bandstructures of the multilayer GNRs. The upper left panel of Fig. 4(a) plots the bandstructure of the monolayer $n=22$ AGNR, which has a width of $W_{\mathrm{GNR}} \approx 2.7 \mathrm{~nm}$ and a band gap of $E_{\mathrm{g}} \approx 516 \mathrm{meV}$. In contrast to a CNT in which a periodic boundary condition applies in the circumferential direction of the $\mathrm{CNT}$ and a valley degeneracy factor of two exists for each band, the monolayer AGNR does not have a valley degeneracy due to a different quantization boundary condition in the width direction of the GNR. At the limit of zero interlayer coupling, an $m$-layer GNR has the same bandstructure as the monolayer, but each band becomes $m$-fold degenerate due to the $m$ uncoupled layers. The interlayer coupling lifts the subband degeneracy. Figure 4(a) also shows the bandstructures of two-, five-, and ten-layer multilayer GNRs with an $\mathrm{AB}$ stacking structure. As the number

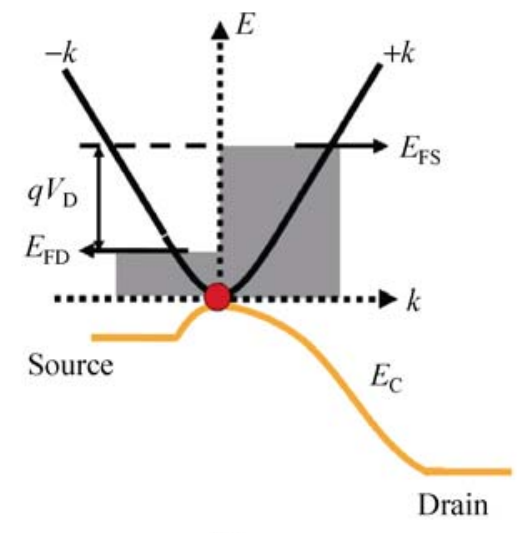

(a)

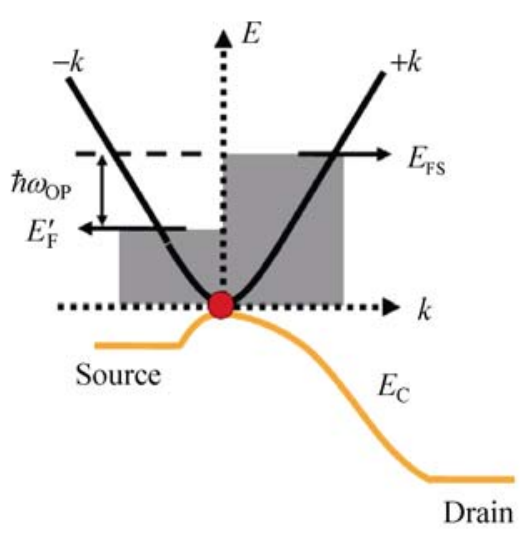

(b)

Figure 3 (a) In the "top of barrier" ballistic transistor model, the $+k$ states are filled according to the source Fermi level $E_{\mathrm{FS}}$, and the $-k$ states are filled according to the drain Fermi level $E_{\mathrm{FD}}$. (b) In the presence of OP scattering, the $-k$ states are filled according to an effective Fermi level, $E_{\mathrm{F}}^{\prime}=E_{\mathrm{FS}}-\hbar \omega_{\mathrm{OP}}$ 

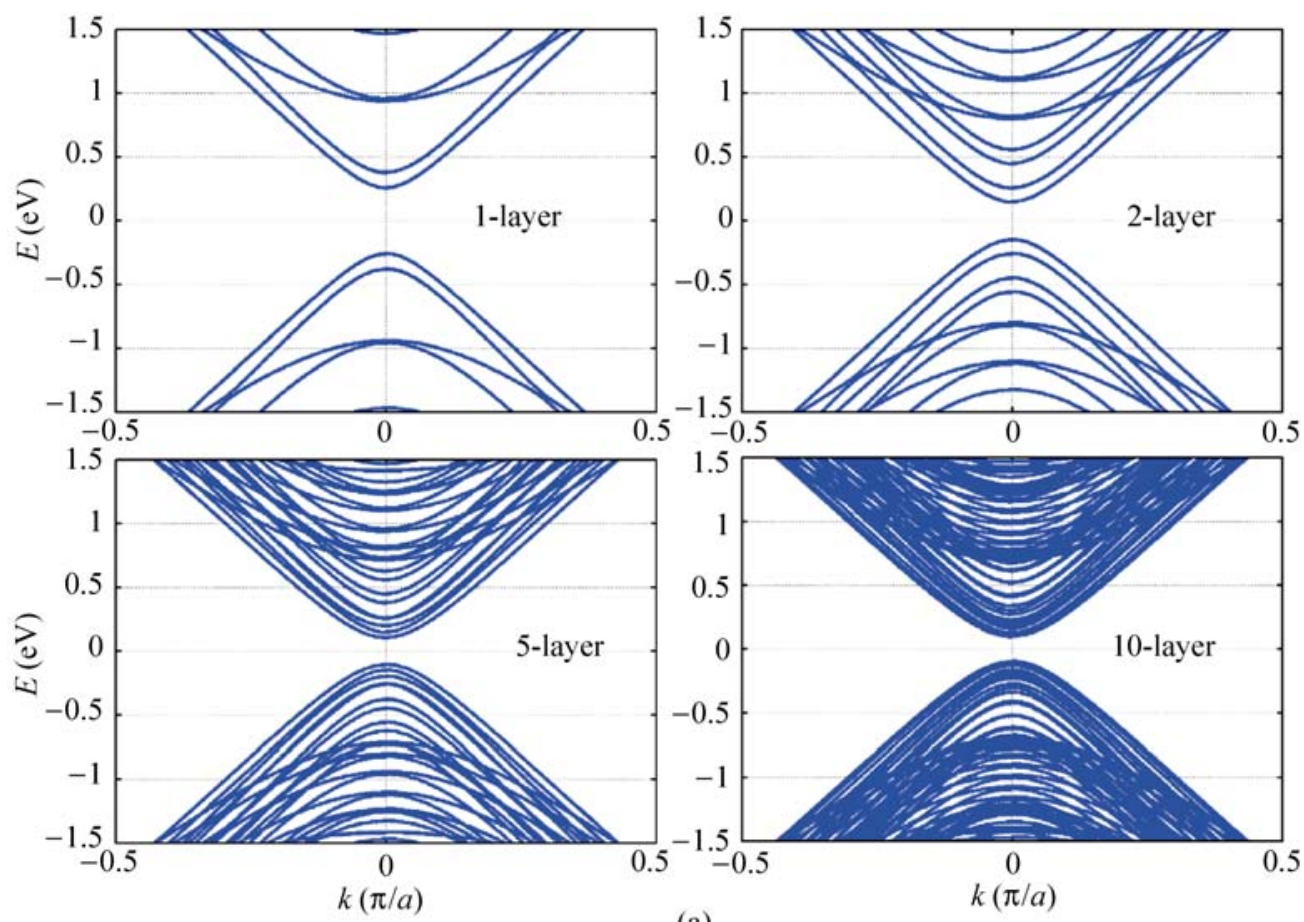

(a)

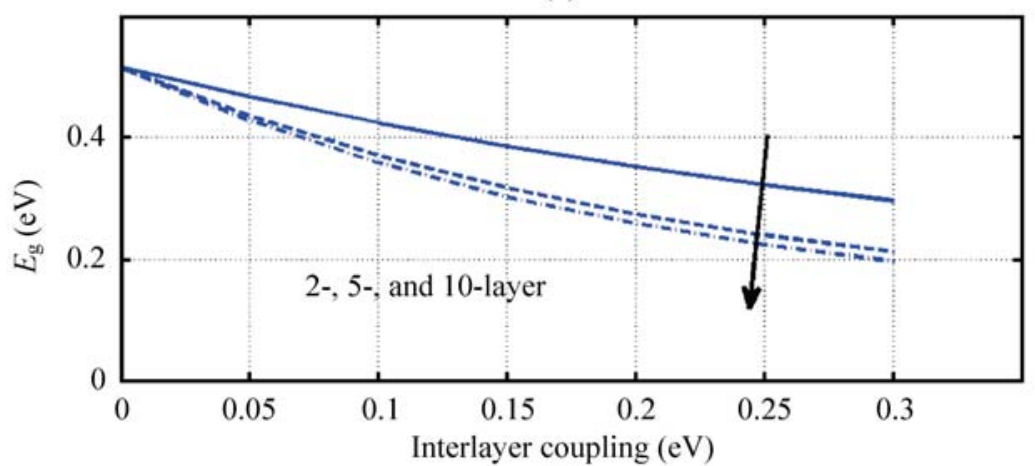

(b)

Figure 4 (a) The bandstructures of one-, two-, five-, and ten-layer $n=22$ multilayer AGNRs with an interlayer coupling of 0.30 eV. The bandgap is $0.52 \mathrm{eV}$ for one-layer, $0.30 \mathrm{eV}$ for two-layer, $0.21 \mathrm{eV}$ for five-layer, and $0.20 \mathrm{eV}$ for ten-layer AGNRs. (b) The bandgap as a function of interlayer coupling for two-layer, five-layer and ten-layer $n=22$ AGNRs

of layers increases, the subband spacing decreases, and the upper subbands should become more accessible for carrier transport. Furthermore, as the layer number increases from one to ten, the bandgap decreases from $516 \mathrm{meV}$ to $197 \mathrm{meV}$. We also examined the dependence of the bandgap on the interlayer coupling strength for the multilayer GNRs as shown in Fig. 4(b). As the interlayer coupling strength increases from the zero limit to the value for the $\mathrm{AB}$ stacking structure, the bandgap decreases monotonically regardless of the number of GNR layers. The decrease is more significant for a multilayer GNR with a larger number of layers.

Next, we examine the effect of gating on transistor performance by varying the $\mathrm{SiO}_{2}$ gate oxide thickness $t_{\mathrm{ox}}$ from $0.23 \mathrm{~nm}$ to $20 \mathrm{~nm}$, as shown in Fig. 5 . A common off-current of $10 \mathrm{nA}$ was specified for all transistors. The lower limit of the simulated $\mathrm{SiO}_{2}$ thickness is equivalent to the 3-nm-thick $\mathrm{ZrO}_{2}$ gate for the modeled gating structure shown in Fig. 1, and results in the same gate insulator capacitance. The on-current increases only slightly by $12 \%$ as the number 
of the layers increases from one to ten at $t_{\mathrm{ox}}=20 \mathrm{~nm}$ as shown in Fig. 5(a). The transistor on-current is determined by the product of the charge density and the average carrier velocity at the top of the channel potential barrier. The relatively small improvement is largely due to a small increase in carrier density as the number of layers increases. The gate to channel capacitance $C_{\mathrm{g}}$ is a serial combination of insulator capacitance $C_{\text {ins }}$ and the quantum capacitance $C_{\mathrm{q}}$, and is limited by $C_{\text {ins }}$ since $C_{\text {ins }}$ is much smaller than $C_{\mathrm{q}}$. A larger number of layers results in a slightly larger $C_{\text {ins }}$ and thereby slightly larger $C_{g}$ due to a thicker channel. For thick oxide gates, the advantage of using a multilayer GNR channel in terms of the on-current is insignificant.

In contrast, the advantage of the multilayer GNR

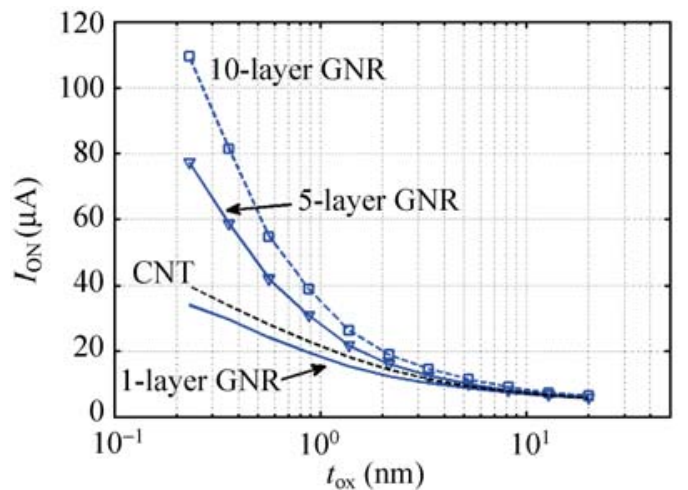

(a) channel in terms of the on-current becomes more significant as $t_{\mathrm{ox}}$ decreases, as shown in Fig. 5(a). Table 1 shows a comparison of the performance of various channels with a high- $\kappa 3 \mathrm{~nm} \mathrm{ZrO}_{2}$. For a common off-current of $10 \mathrm{nA}$, the monolayer GNR channel has a slightly larger gate insulator capacitance, but a $13 \%$ smaller on-current than the CNT channel, because of the lack of valley degeneracy in the monolayer GNR. As the number of layers increases, the on-current surpasses that of the CNT. For a five-layer GNR, the on-current is $97 \%$ larger than that of the $\mathrm{CNT}$, and for a ten-layer GNR, the on-current is $180 \%$ larger than that of the CNT. For a similar gating, the ballistic on-current of the multilayer GNR can be significantly larger than that of a CNT with a similar bandgap.

In order to understand the factors that contribute

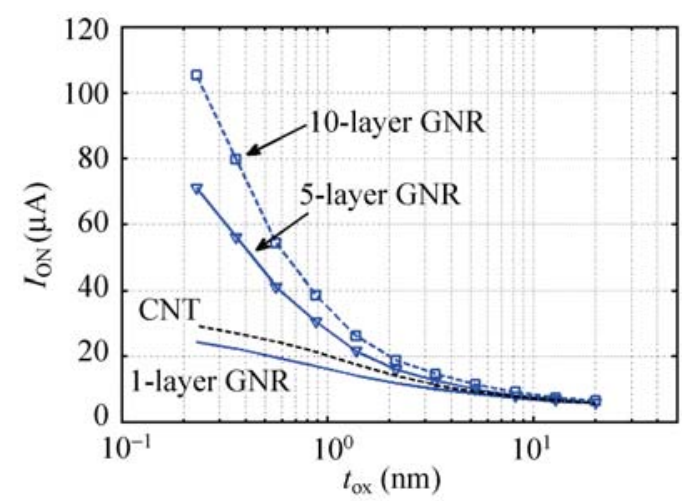

(b)

Figure 5 Effect of the gate insulator. The on-current as a function of the gate oxide thickness (a) at the ballistic limit and (b) in the presence of OP scattering for the GNRFETs and CNTFETs shown in Fig. 1. The one-, five-, and ten-layer $n=22$ AGNRs with a zero interlayer coupling have a similar bandgap to the simulated $(20,0) \mathrm{CNT}$ with a $E_{\mathrm{g}, \mathrm{CNT}} \approx 0.50 \mathrm{eV}$ to ensure a fair comparison. The gate insulator dielectric constant is $\kappa_{\mathrm{SiO}_{2}} \approx 3$.9. The smallest simulated value of the oxide thickness, $t_{\mathrm{ox}} \approx 0.23 \mathrm{~nm}$, is the equivalent value for a 3-nm thick $\mathrm{ZrO}_{2}$ gate insulator with a dielectric constant of $\kappa \approx 25$, which results in the same gate insulator capacitance

Table 1 Comparison of the $(20,0)$ CNTFET with one-, five-, and ten-layer $n=22$ GNRFETs at the ballistic performance limits. The transistor structures are shown in Fig. 1. The gate insulator thickness is $3 \mathrm{~nm}$ and the dielectric constant is 25 (for $\mathrm{ZrO}_{2}$ ). The off-current $\left(I_{\mathrm{OFF}}\right)$, gate insulator capacitance $\left(C_{\mathrm{ins}}\right)$, on-current $\left(I_{\mathrm{ON}}\right)$, spacing between the source Fermi level and the top of the first subband barrier $\left(E_{\mathrm{FS}}-E_{1}\right)$, on-off current ratio $\left(I_{\mathrm{ON}} / I_{\mathrm{OFF}}\right)$, and average carrier velocity at the top of the barrier $(\langle v(0)\rangle)$ are compared. For the modeled one-layer GNR, the spacing between the first and second subbands is $E_{2}-E_{1}=0.121 \mathrm{eV}$. For the modeled CNT, $E_{2}-E_{1}=0.226 \mathrm{eV}$

\begin{tabular}{ccccccc}
\hline & $I_{\mathrm{OFF}}(\mathrm{nA})$ & $C_{\mathrm{ins}}(\mathrm{F} / \mathrm{m})$ & $I_{\mathrm{ON}}(\mu \mathrm{A})$ & $E_{\mathrm{FS}}-E_{1}(\mathrm{eV})$ & $I_{\mathrm{ON}} / I_{\mathrm{OFF}}$ & $\langle v(0)\rangle(\mathrm{cm} / \mathrm{s})$ \\
\hline CNT & 10 & $8.42 \times 10^{-10}$ & 39.9 & 0.233 & 3900 & $4.28 \times 10^{7}$ \\
One-layer GNR & 10 & $8.98 \times 10^{-10}$ & 34.1 & 0.279 & 3400 & $4.61 \times 10^{7}$ \\
Five-layer GNR & 10 & $1.27 \times 10^{-9}$ & 77.3 & 0.155 & 7700 & $3.69 \times 10^{7}$ \\
Ten-layer GNR & 10 & $1.73 \times 10^{-9}$ & 109.6 & 0.120 & 11000 & $3.50 \times 10^{7}$ \\
\hline
\end{tabular}


to the increase in the on-current, we also computed the gate insulator capacitance and the average carrier velocity as shown in Table 1 . The average carrier velocity decreases from $4.28 \times 10^{7} \mathrm{~cm} / \mathrm{s}$ to $3.50 \times 10^{7} \mathrm{~cm} / \mathrm{s}$ as the number of GNR layers $m$ increases from one to ten because more carriers populate closer to the bottom of the $m$-fold degenerate lowest subbands where the band-structure-limited velocity is low. The on-current, however, increases by a factor of 3.2 due to the increase in the gate capacitance $C_{\mathrm{g}}$ by a factor of 3.9. It is also interesting to note that the increase in the gate capacitance significantly outpaces the increase in the gate insulator capacitance stemming from a thicker GNR body as the number of GNR layers increases, because of the proportional increase in the quantum capacitance as a function of the number of the layers. Furthermore, for the modeled thin high- $\kappa$ gate insulator, the quantum capacitance and the gate insulator capacitance become comparable, and both of them play a role in determining the gate capacitance.

The improvement in on-current is even more significant in the presence of OP scattering. As shown in Table 2, the on-current increases even more significantly as the number of layers increases in the presence of OP scattering. The on-current of the ten-layer GNR channel is $260 \%$ larger than that of the CNT. In addition, the average carrier velocity is also larger, which is different from the case of the ballistic channel as shown in Table 1. For a CNTFET, the OP scattering results in a saturation current close to $\left(4 e^{2} / h\right) \times\left(\hbar \omega_{\mathrm{OP}}\right) \approx 28 \mu \mathrm{A}$, where the OP energy $\hbar \omega_{\mathrm{OP}} \approx$ $180 \mathrm{meV}$ when only the first subband conducts the current, which is the case for the CNTFET because $E_{\mathrm{FS}}-E_{1} \approx 216 \mathrm{meV}$ is smaller than the spacing between the first and second subbands $E_{2}-E_{1} \approx 226 \mathrm{meV}$. The OP scattering results in considerably increased population of the $-k$ states, which also lowers the average carrier velocity at the top of the barrier from
$4.61 \times 10^{7} \mathrm{~cm} / \mathrm{s}$ to $2.72 \times 10^{7} \mathrm{~cm} / \mathrm{s}$. In contrast, the effect of OP scattering on the ten-layer GNRFET is small, because the value of $E_{\mathrm{FS}}-E_{1} \approx 120 \mathrm{meV}$ is smaller than the OP energy. In a multilayer GNR, more subbands are responsible for delivering the on-current, and therefore, the $E_{\mathrm{FS}}-E_{1}$ value is smaller. As a result, the OP scattering only has a small effect on both the on-current and average carrier velocity of the ten-layer GNRFET. In constrast to the case of the ballistic channel, the average carrier velocity of the ten-layer GNRFET is also about 20\% larger than that of the CNTFET in the presence of OP scattering, which promises faster intrinsic transistor speeds.

Finally, we examine the dependence of the oncurrent on the interlayer coupling. As shown in Fig. 6, the largest on-current is achieved at zero interlayer coupling and the on-current decreases as the interlayer coupling increases. The ballistic on-current monotonically decreases from $51 \mu \mathrm{A}$ to $36 \mu \mathrm{A}$ for two layers and from $77 \mu \mathrm{A}$ to $58 \mu \mathrm{A}$ for five layers as the interlayer coupling increases to the value for $A B$ stacking. The reason is that an increase in the interlayer coupling increases the spacing between the subbands, which makes the higher subbands more difficult to be accessed for delivering the on-current. In addition, the increase in interlayer coupling also decreases the bandgap, as shown in Fig. 4. As the bandgap decreases, the band-to-band tunneling can be turned on, which can significantly increase the offcurrent. (Modeling the band-to-band tunneling current is beyond the capability of the semiclassical model used here.) Non-AB-stacking multilayer structures have been recently observed in experiments for both GNRs unzipped from CNTs [13] and CVD-grown multilayer graphene structures. Weakening the interlayer coupling by non-AB-stacking, such as in randomly stacking structures, should be pursued in order to boost the performance of multilayer GNRFETs.

Table 2 The same comparison as Table 1 in the presence of OP scattering

\begin{tabular}{ccccccc}
\hline With OP & $I_{\mathrm{OFF}}(\mathrm{nA})$ & $C_{\mathrm{ins}}(\mathrm{F} / \mathrm{m})$ & $I_{\mathrm{ON}}(\mu \mathrm{A})$ & $E_{\mathrm{FS}}-E_{1}(\mathrm{eV})$ & $I_{\mathrm{ON}} / I_{\mathrm{OFF}}$ & $\langle v(0)\rangle(\mathrm{cm} / \mathrm{s})$ \\
\hline CNT & 10 & $8.42 \times 10^{-10}$ & 29.3 & 0.216 & 2900 & $2.72 \times 10^{7}$ \\
One-layer GNR & 10 & $8.98 \times 10^{-10}$ & 24.4 & 0.260 & 2400 & $2.69 \times 10^{7}$ \\
Five-layer GNR & 10 & $1.27 \times 10^{-9}$ & 71.0 & 0.151 & 7100 & $3.30 \times 10^{7}$ \\
Ten-layer GNR & 10 & $1.73 \times 10^{-9}$ & 105.4 & 0.118 & 10500 & $3.33 \times 10^{7}$ \\
\hline
\end{tabular}




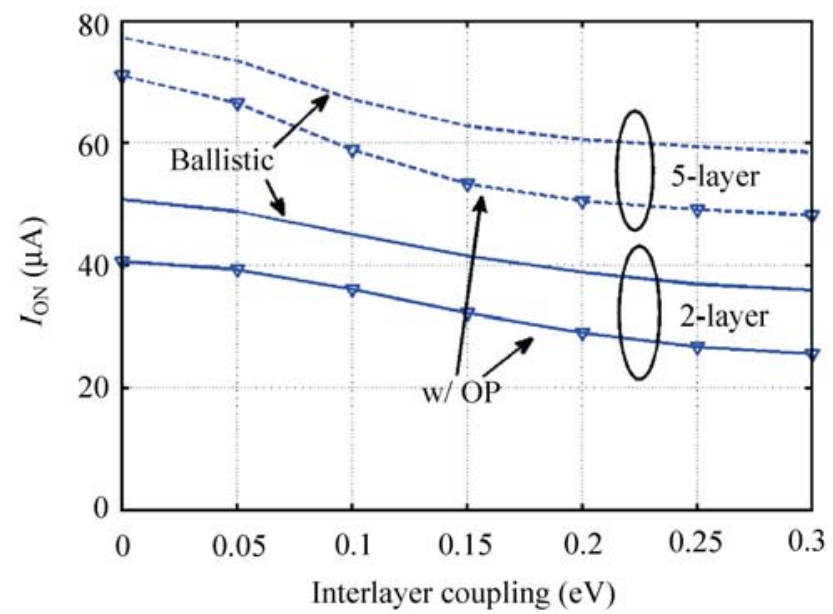

Figure 6 Effect of interlayer coupling. The on-current as a function of the interlayer coupling for the five-layer GNRFET (dashed lines) and the two-layer GNRFET (solid lines) as shown in Fig. 1. A 3-nm-thick $\mathrm{ZrO}_{2}$ gate insulator is used. The lines without $\nabla$ symbols are computed for a ballistic channel and the lines with $\nabla$ symbols corrrespond to the presence of OP scattering

\section{Discussions and conclusions}

The performance of multilayer GNR MOSFETs can also be compared with that of silicon MOSFETs. Such a comparison is clouded by the different dimensionality of the channel material, and here we simply discuss how dense a GNR array channel needs to be to meet the performance goal at the end of the International Technology Roadmap for Semiconductors (ITRS roadmap) [19]. The ITRS roadmap calls for an oncurrent of about $2700 \mu \mathrm{A} / \mu \mathrm{m}$ and an off-current of $0.60 \mu \mathrm{A} / \mu \mathrm{m}$ at a power supply voltage of $0.65 \mathrm{~V}$ for the technology nodes near year 2020. To reach an on-current of $2700 \mu \mathrm{A} / \mu \mathrm{m}$, a ten-layer array GNRFET with 26 GNRs per $\mu \mathrm{m}$ is needed as shown in Table 2 . The array has an off-current of $0.26 \mu \mathrm{A} / \mu \mathrm{m}$, which is less than half of the ITRS goal, although the power supply used in the GNRFET simulation is only $0.5 \mathrm{~V}$. A recent experimental study characterized the mobility degradation of monolayer graphene on a $\mathrm{SiO}_{2}$ substrate [20]. Another experimental study showed that a multilayer graphene transistor was more immune to noise compared with a monolayer graphene transistor [21], whose one-atom-thick body was susceptible to the charge impurities and oxide traps near the transistor channel. Therefore, the multilayer GNRFET is expected to have a better immunity to the adverse effects from substrates than the monolayer GNRFET.

The bandstructure of a multilayer GNR was assumed to be gate-voltage-independent in this study. Large electric fields and a significant potential drop between the layers, however, can alter the bandstructure of a multilayer GNR. For the simulated gate-all-around structure and a low applied voltage below $0.5 \mathrm{~V}$, the electric field between the layers and the resulting potential drop between the layers is small (less than $30 \mathrm{meV}$ as computed by a separate self-consistent atomistic simulation as described in Refs. [22, 23]). Its effect on the transistor $I-V$ characteristics is negligible for the modeled gating structure and power supply voltage.

In summary, we have shown the important role of developing good gating technology and weakening the interlayer coupling in improving the performance of multilayer GNRFETs. The thin high- $\kappa$ gate insulator is already in production for the Intel $45 \mathrm{~nm}$ transistor, but its application to GNRFETs remains to be developed. Weakening the interlayer coupling could be achieved experimentally in non-AB-stacking multilayer graphene. The performance limits of a welldesigned multilayer GNRFET can significantly exceed its CNT counterpart with a similar gate and bandgap in terms of the on-current, at either the ballistic limit or in the presence of OP scattering.

\section{Acknowledgements}

This work was supported by the National Science Foundation (NSF) and the Office of Naval Research (ONR), Intel, and MARCO MSD.

\section{References}

[1] Novoselov, K. S.; Geim, A. K.; Morozov, S. V.; Jiang, D.; Zhang, Y.; Dubonos, S. V.; Grigorieva, I. V.; Firsov, A. A. Electric field effect in atomically thin carbon films. Science 2004, 306, 666-669.

[2] Zhang, Y.; Tan, Y.; Stormer, H. L.; Kim, P. Experimental observation of the quantum Hall effect and Berry's phase in graphene. Nature 2005, 438, 201-204. 
[3] Berger, C.; Song, Z.; Li, X.; Wu, X.; Brown, N.; Naud, C.; Mayou, D.; Li, T.; Hass, J.; Marchenkov, A. N.; Conrad, E. H.; First, P. N.; de Heer, W. A. Electronic confinement and coherence in patterned epitaxial graphene. Science 2006, 312, 1191-1196.

[4] Li, X.; Wang, X.; Zhang, L.; Lee, S.; Dai, H. Chemically derived, ultrasmooth graphene nanoribbon semiconductors. Science 2008, 319, 1229-1232.

[5] Lin, Y.; Perebeinos, V.; Chen, Z.; Avouris, P. Electrical observation of subband formation in graphene nanoribbons. Phys. Rev. B 2008, 78, 161409.

[6] Wang, X.; Ouyang, Y.; Li, X.; Wang, H.; Guo, J.; Dai, H. Room-temperature all-semiconducting sub-10-nm graphene nanoribbon field-effect transistors. Phys. Rev. Lett. 2008, 100, 206803.

[7] Ouyang, Y.; Wang, X.; Dai, H.; Guo, J. Carrier scattering in graphene nanoribbon field-effect transistors. Appl. Phys. Lett. 2008, 92, 243124.

[8] Gunlycke, D.; Lawler, H. M.; White, C. T. Roomtemperature ballistic transport in narrow graphene strips. Phys. Rev. B 2007, 75, 085418.

[9] Ouyang, Y.; Yoon, Y.; Fodor, J. K.; Guo, J. Comparison of performance limits for carbon nanoribbon and carbon nanotube transistors. Appl. Phys. Lett. 2006, 89, 203107.

[10] Rahman, A.; Jing Guo; Datta, S.; Lundstrom, M. Theory of ballistic nanotransistors. IEEE Trans. Electron Dev. 2003, 50, 1853-1864.

[11] Chen, Z.; Appenzeller, J.; Knoch, J.; Lin, Y.; Avouris, P. The role of metal-nanotube contact in the performance of carbon nanotube field-effect transistors. Nano Lett. 2005, 5, 1497-1502.

[12] Javey, A.; Tu, R.; Farmer, D. B.; Guo, J.; Gordon, R. G.; Dai, H. High performance n-type carbon nanotube fieldeffect transistors with chemically doped contacts. Nano Lett.
2005, 5, 345-348.

[13] Jiao, L.; Zhang, L.; Wang, X.; Diankov, G.; Dai, H. Narrow graphene nanoribbons from carbon nanotubes. Nature 2009, $458,877-880$.

[14] Kosynkin, D. V.; Higginbotham, A. L.; Sinitskii, A.; Lomeda, J. R.; Dimiev, A.; Price, B. K.; Tour, J. M. Longitudinal unzipping of carbon nanotubes to form graphene nanoribbons. Nature 2009, 458, 872-876.

[15] Nakada, K.; Fujita, M.; Dresselhaus, G.; Dresselhaus, M. S. Edge state in graphene ribbons: Nanometer size effect and edge shape dependence. Phys. Rev. B 1996, 54, 17954.

[16] Son, Y.; Cohen, M. L.; Louie, S. G. Energy gaps in graphene nanoribbons. Phys. Rev. Lett. 2006, 97, 216803.

[17] Grüneis, A.; Attaccalite, C.; Wirtz, L.; Shiozawa, H.; Saito, R.; Pichler, T.; Rubio A. Tight-binding description of the quasiparticle dispersion of graphite and few-layer graphene. Phys. Rev. B 2008, 78, 205425.

[18] Yao, Z.; Kane, C. L.; Dekker, C. High-field electrical transport in single-wall carbon nanotubes. Phys. Rev. Lett. 2000, 84, 2941-2944.

[19] ITRS, International Technology Roadmap for Semiconductors, http://www.itrs.net (accessed oct 2, 2009).

[20] Chen, J.; Jang, C.; Xiao, S.; Ishigami, M.; Fuhrer, M. S. Intrinsic and extrinsic performance limits of graphene devices on $\mathrm{SiO}_{2}$. Nat. Nanotechnol. 2008, 3, 206-209.

[21] Sui, Y.; Appenzeller, J. Screening and interlayer coupling in multilayer graphene field-effect transistors. Nano Lett. 2009, 9, 2973-2977.

[22] Guo, J.; Yoon, Y.; Ouyang, Y. Gate electrostatics and quantum capacitance of graphene nanoribbons. Nano Lett. 2007, 7, 1935-1940.

[23] Neophytou, N.; Paul, A.; Lundstrom, M.; Klimeck, G. Bandstructure effects in silicon nanowire electron transport. IEEE Trans. Electron Dev. 2008, 55, 1286-1297. 